Mini Review

\title{
Hypermethylation and Hypomethylation of DNA: Implication for Diagnosis and Prognosis of Prostate Cancer
}

\author{
${ }^{1,2}$ Lubos Danisovic and ${ }^{3}$ Stanislav Ziaran \\ ${ }^{1}$ Institute of Medical Biology, Genetics and Clinical Genetics, Faculty of Medicine, University of the Comenius, \\ Bratislava, Slovakia \\ ${ }^{2}$ Regenmed Ltd., Bratislava, Slovakia \\ ${ }^{3}$ Department of Urology, Faculty of Medicine and University Hospital, Comenius University, Bratislava, Slovakia
}

Article history

Received: 03-01-2015

Revised: 01-06-2015

Accepted: 08-06-2015

Corresponding Author:

Lubos Danisovic

Institute of Medical Biology,

Genetics and Clinical Genetics,

Faculty of Medicine, Comenius

University, Bratislava, Slovakia

Email: lubos.danisovic@fmed.uniba.sk

\begin{abstract}
Prostate cancer belongs to the most common cancers and it is the second leading cause of cancer death in men. A genetic predisposition with epigenetic changes in DNA contributes to the development of the disease. DNA hypermethylation and hypomethylation belong to the most studied epigenetic changes in prostate cancer development. Both forms may lead to chromosomal instability and transcriptional gene silencing. This article is aimed at brief review of information in respect to DNA hypermethylation and hypomethylation in the prostate cancer development. Moreover, we discuss their implication for diagnosis and prognosis of prostate cancer.
\end{abstract}

Keywords: Prostate Cancer, DNA Hypermethylation, DNA Hypomethylation, Diagnosis, Prognosis

\section{Introduction}

Prostate Cancer $(\mathrm{PCa})$ belongs to the most common cancers and it is the second leading cause of cancer death in men (over 250.000 men dies worldwide every year). A genetic predisposition or acquired genetic and epigenetic changes with the effect of other factors, such as advanced age, race, diet, weight, physical activity, endogenous hormones and environmental factors contribute to $\mathrm{PCa}$ development (Demichelis and Stanford, 2014).

$\mathrm{PCa}$ is a very heterogeneous disease that is characterized by different clinical behavior, from indolent, slow-growing tumors to aggressive, fastgrowing tumors with lethal progression. Early diagnostics and identification of PCa type are crucial prerequisites for efficient treatment of patients. Recently, the diagnostics of early stages of $\mathrm{PCa}$ is based mostly on evaluation of Prostate-Specific Antigen (PSA) in serum of patients (Lilja et al., 2008). Men with high levels of PSA undergo biopsy in order to determine histopatological grading of PCaGleason scoring which classifies tumors from 1 to 5 (most to least differentiated) (Epstein, 2010) as well as staging-determination of the status of their primary tumors (T1-T4; from bounded to fully invasive), with or without lymph node involvement (N0 or 1) (Cheng et al., 2012). The results from this screening diagnosis lead into conventional treatment, including radical prostatectomy and brachytherapy. In case of advanced $\mathrm{PCa}$, conventional treatment continues with androgen deprivation therapy. However, in many cases the cancer recurs (Michaelson et al., 2008).

At present, the clinicians and researchers are forced to find more precise and sensitive biomarker suitable for $\mathrm{PCa}$ diagnostics as well as prognostics and therapy. The excessive study of epigenetical mechanisms, including DNA hypermethylation and hypomethylation provides promising results in this context (Chin et al., 2011).

The main goal of the present article is to offer brief review of information in respect to DNA hypermethylation and hypomethylation and their implications for $\mathrm{PCa}$ development, diagnosis and prognosis.

\section{DNA Methylation in Prostate Cancer}

Methylation of the DNA is its covalent modification by binding of the methyl group to the cytosine in dinucleotides containing Cytosine and Guanine $(\mathrm{CpG})$. These dinucleotides are present in the DNA sequence and they are termed as $\mathrm{CpG}$ islands. They occur in about $60 \%$ of all genes in the human genome. They are responsible for controlling the gene expression and their 
methylation lead into silencing of the transcriptional activity of the downstream genes (Irizarry et al. 2009). There are a lot of different genes, including tumor suppressor genes, genes involved in metabolism and genes responsible for cell invasion, which has been extensively studied in respect to cancer development and progression including PCa due to their methylation (Yang and Park, 2012). Aberrant DNA methylation can occur in two formshypermethylation and hypomethylation.

\section{Hypermethylation}

The most known genome alteration in $\mathrm{PCa}$ is hypermethylation of $\mathrm{CpG}$ at promoter of Glutathione $\mathrm{S}$ Transferase P1 (GSTP1). It occurs in majority ( $>90 \%)$ of PCa cases but it is not present in normal tissues (Lee et al., 1997). The lost of GSTP1 activity lead into increasing of oxidative damage of DNA which may lead in increase of PCa risk. GSTP1 hypermethylation is usually detected in high frequency in urine, blood and ejaculates of PCa patients.

Another frequently investigated is gene for Tissue Inhibitor of Metalloproteinase-2 (TIMP-2) which is involved in $\mathrm{PCa}$ progression and metastasis. It was documented that hypermethylation of gene for TIMP-2 lead into its down-expression in $\mathrm{PCa}$ when compared with normal tissue (Pulukuri et al., 2007). On the contrary, there are several works which described the overexpression of TIMP-2 in PCa. Moreover, Ross et al. (2003) showed correlation between TIMP-2 expression and clinical stages and recurrence.

The lack of Retinoic Acid Receptor $\beta$ (RAR $\beta)$ expression is typical for tumor tissues. This is due to hypermethylation of promoter RAR $\beta$ which occurs in 40 $-84 \%$ of PCs tissues (Nakayama et al., 2001; Zon et al., 2009). Moreover, moderate or high frequencies of RAR $\beta$ promoter methylation were also documented in urine or blood samples (Bastian et al., 2008; Rouprêt et al., 2008). It makes RAR $\beta$ promoter hypermethylation a promising biomarker for early PCa detection.

Recent studies, reported hypermethylation in promoter of Caveolin-1 (CAV1) gene in respect to $\mathrm{PCa}$. $C A V 1$ belong to tumors suppressor genes which are involved in vesicular transport, cholesterol balance, transformation and tumorigenesis. Moreover, it acts as metastasis-promoting gene (Chang et al., 2013; Patra and Bettuzzi, 2007). It was shown that increased DNA hypermethylation of CAV1 gene is associated with biochemical recurrence in patients with $\mathrm{PCa}$ (Bachmann et al., 2008). On other hand CAV1 overexpression was recorded in patients with aggressive PCa recurrence (Karam et al., 2007). These conflicting data suggest that hypermethylation of $C A V 1$ may not be unfailing biomarker for detection of PCa.
The Table 1 provides overview of different genes which has been studied in respect to hypermethylation and PCa development.

\section{Hypomethylation}

DNA hypomethylation or demethylation can lead to structural and functional alternations of the genome. There are two forms of DNA hypomethylation-global (also known as genomic) and localized (also known as gene-specific) (Li et al., 2005).

Global hypomethylation is associated with reduced 5methylcytosine content in the genome. For example, in case of some adenocarcinomas the genomic content of 5methylcytosine is decreased by an average of $10 \%$. Global hypomethylation was also reported in the premalignant or early stages of some neoplasia (Barciszewska et al., 2014; Ehrlich, 2009).

The pioneer work by (Bedford and van Helden, 1987) showed decreased content of 5-methylcytosine in patients with benign prostatic hyperplasia and metastatic tumors in comparison with non-metastatic prostate tumors. More recently, it was demonstrated that the repetitive DNA elements LINE-1 and SAT2 are heavily methylated in normal cells, but methylation levels of these elements in PCa cells are significantly lower (Cho et al., 2009).

Localized hypomethylation is related to a significant decrease in cytosine methylation level. It was demonstrated that some genes are substantially hypomethylated in cancer cells, but not in normal cells (Hammoud et al., 2013). Moreover, compared with normal cells, cancer cells possess two hypomethylated rasoncogenes, c-Ha-ras and c-Ki-ras and also c-jun and c-MYC protooncogenes (Li et al., 2005).

In PCa, PLAU gene responsible for expression urokinase plasminogen activator (protein involved in promotion the tumor invasion and metastasis) is hypomethylated (Banyard et al., 2014). Other hypomethylated genes in PCa are CAGE (a novel cancer/testis antigen gene) and heparanase. A methylationspecific PCR analysis revealed that hypomethylation of the CAGE promoter was present at frequency about $40 \% \mathrm{PCa}$, but not in normal prostate cells (Cho et al., 2003). It was also shown that heparanase (endo- $\beta$-D-glucuronidase) is overexpresed in $\mathrm{PCa}$ cells due to its substantial hypomethylation (Ogishima et al., 2005).

More recently, it was demonstrated that several other genes, including histone methyltransferase Enhancer of Zeste Homologue 2 (EZH2), Melanoma Antigen Gene Protein-A11 (MAGE-11), nsulin-like Growth Factor-2 (IGF2), Cancer Testis Antigene (CTA) etc., are hypomethylated in PCa cells (Ribarska et al., 2012; Bhusari et al., 2011; Karpf et al., 2009; Yegnasubramanian et al. 2008). 
Table 1. Overview of different genes studied in respect to hypermethylation and PCa development

\begin{tabular}{ll}
\hline Group & Examples \\
\hline Tumor suppressor & $\begin{array}{l}\text { Caveolin-1 Cyclin-dependent } \\
\text { kinase inhibitors Death-associated } \\
\text { protein kinase Fragile histidine } \\
\text { triad Lipoprotein lipase }\end{array}$ \\
& $\begin{array}{l}\text { Prostaglandin-endoperoxide } \\
\text { synthase 2Tumor necrosis factor } \\
\text { receptor superfamily }\end{array}$ \\
& Androgen receptor estrogen \\
receptors Retinoic acid receptor $\beta$ \\
menes involved in & Glutathione S transferase P1 \\
& Cellular retinol-binding protein 1 \\
& Endothelin B receptor Gene \\
& Aldehyde dehydrogenase 1A2 and 1A3 \\
& Adenomatous polyposis coli (APC) \\
CD44 & E-cadherin \\
Tumor cell & H-cadherin \\
invasion / metastasis & S100 calcium-binding protein A2 \\
& Tissue inhibitor of metalloproteinase-2 \\
& SRC family tyrosine kinase \\
& Neutral endopeptidase 24.11 \\
& Methylguanine-methyltransferase
\end{tabular}

\section{Methylation of DNA as Prostate Cancer Biomarker}

The "classical" diagnostics of PCa is based mainly on evaluation of PSA levels in serum and evaluation of needle biopsies. In some cases the results may be false negative. Recently, these standard approaches are supplemented by molecular diagnostics, including evaluation of DNA methylation, which has several advantages. First, it always occurs in defined DNA regions and can be detected using techniques of high sensitivity and resolution (e.g., methylation-specific PCR, bisulfite genomic sequencing). Second, hypermethylation occurs in every type of tumor. These characteristics make DNA methylation and superior biomarker for detection of cancer, including PCa (Li et al., 2005).

It was also shown that the utilization multigene methylation panel including GSTP1, APC, TIG1 and RAR $\beta 2$ significantly increases the sensitivity of diagnostics of $\mathrm{PCa}$ in comparison with histological evaluation (Rosenbaum et al., 2005).

Especially hypermethylation in GSTP1 have significant importance for $\mathrm{PCa}$ diagnosis and prognosis. For example, Goessl et al. (2001) evaluated DNA hypermethylation in GSTP1 in urine after prostate massage and detected $\mathrm{PCa}$ with a specificity of $98 \%$ and overall sensitivity of $73 \%$. Later, (Woodson et al., 2008) evaluated GSTP1 hypermethylation in urine collected after prostatic massage and in core needle biopsies from 100 men referred for diagnostic biopsy. Methylation of GSTP1 in urine specimens had $75 \%$ sensitivity and $98 \%$ specificity for prostate cancer. GSTP1 methylation in the biopsy had $88 \%$ specificity and $91 \%$ sensitivity. Interestingly they observed a higher frequency of GSTP1 methylation in the urine of men with stage III vs. II disease. In further study, Richiardi and coauthors used hypermethylation analysis not only in GSTP1 but also in APC and RUNX3. They showed that promoter methylation in APC is good marker of $\mathrm{PCa}$ progression and hyperethylation in RUNX3 is associated with prostate cancer mortality. On the contrary hypermethylation in GSTP1 was positively associated with Gleason score but did not predict mortality of patients (Richiardi et al., 2009).

It was also shown that by assessment of DNA hypermethylation it is also possible to distinguish primary $\mathrm{PCa}$ from benign prostate tissues, mainly when analyzed methylation pattern of multiple genes, such as GSTP1, RASSF1, APC, MDR1, PTGS2 and CD44 (Costa-Pinheiro et al., 2014; Kang et al., 2004).

Kollermann et al. (2003) demonstrated utilization of methylation in GSTP1 in detection of occult PCa cells in lymph nodes.

Yang et al. (2013) analyzed global DNA hypomethylation in human $\mathrm{PCa}$ and prostatic intraepithelial neoplasm tissues. They found that global DNA hypomethylation was low in most PCa compared with benign regions from the same individuals. Moreover, their results did not prove association of DNA hypomethylation in primary cancers with pathologic grade and clinical prognosis. On other hand, their results suggest that global DNA hypomethylation might play a pivotal role in the process of $\mathrm{PCa}$ initiation. These results were in stark contrast to finding obtained by (Yegnasubramanian et al., 2008) who provided evidence that DNA hypomethylation (mainly in LINE1 gene) occur not only in primary $\mathrm{PCa}$ but also later during or after metastatic dissemination.

For a more exact interpretation of the results there are available several scoring systems, such as Methylation Index (MI) which is defined as the ratio of methylated genes and the total number of analyzed genes (Tilandyová et al., 2010). The positive correlation betveen MI and Gleason score was demonstrated. It was also demonstrated that increased level of methylation in APC promoter seem to be good predictor of unfavorable prognosis. The higher level of methylation of GSTP1 and APC are in good correlation with high level of PSA. Moreover, hypermethylation of two genes, including GSTP1 and APC correlated with $\mathrm{pT}$ stage or Gleason score. The increased levels of their hypermethylation were also associated with progression of PCa and lead into biochemical recurrence after radical prostatectomy (Ellinger et al., 2008).

On the other hand, the utilization of hypermethylation and hypomethylation of selected genes 
in clinical practice may be affected by biological variability (in selected population) as well as by preparation of material and methological approaches; but this may be overlapped by unification and standardization of selected methods.

\section{Conclusion}

A number of studies were established to check the capability of quantitative changes in DNA methylation, as measured either in $\mathrm{PCa}$ tissues or in serum and urine, to augment prediction of outcome for PCa patients. Although large trials are required prior clinical implementation, several of these studies suggest that hypermethylation and hypomethylation biomarkers may add value to existing models used for prediction of outcome in PCa.

\section{Acknowledgement}

The present work was supported by the grant VEGA No. 1/1250/12.

\section{Author's Contributions}

Lubos Danisovic: Wrote and revised manuscript. Stanislav Ziaran: Wrote and revised manuscript.

\section{Ethics}

Both authors declare that there is no conflict of interest.

\section{References}

Bachmann, N., J. Haeusler, M. Luedeke, K. Rainer and P. Sven et al., 2008. Expression changes of $C A V_{l}$ and $\mathrm{EZH}_{2}$, located on $7 \mathrm{q} 31$ approximately q36, are rarely related to genomic alterations in primary prostate carcinoma. Cancer Genet. Cytogenet., 182:103-110. DOI: 10.1016/j.cancergencyto.2008.01.006

Banyard, J., I. Chung, M. Migliozzi, T.P. Derek and M.W. Arianne et al., 2014. Identification of genes regulating migration and invasion using a new model of metastatic prostate cancer. BMC Cancer, 14: 387-387. DOI: 10.1186/1471-2407-14-387

Barciszewska, A.M., S. Nowak and M.Z. NaskrętBarciszewska, 2014. The degree of global DNA hypomethylation in peripheral blood correlates with that in matched tumor tissues in several neoplasia. PLoS ONE, 9: e92599.

DOI: 10.1371/journal.pone.0092599

Bastian, P.J., G.S. Palapattu, S. Yegnasubramanian, G.R. Craig and L. Xiaohui et al., 2008. CpG island hypermethylation profile in the serum of men with clinically localized and hormone refractory metastatic prostate cancer. J Urol., 179: 529-34. DOI: 10.1016/j.juro.2007.09.038
Bedford, M.T. and P.D. Van Helden, 1987. Hypomethylation of DNA in pathological conditions of the human prostate. Cancer Res., 47: 5274-5276. PMID: 2443238

Bhusari, S., B. Yang, J. Kueck, H. Wei and F. David et al., 2011. Insulin-like growth factor-2 (IGF2) loss of imprinting marks a field defect within human prostates containing cancer. Prostate, 71: 1621-30. DOI: $10.1002 /$ pros.21379

Chang, W.S., S.S. Lin, F.J. Li, C.W. Tsai and L.Y. Li et al., 2013. Significant association of caveolin-1 (CAVI) genotypes with upper urothelial tract cancer. Anticancer Res., 33: 4907-4912. PMID: 24222128

Cheng, L., R. Montironi, D.G. Bostwick, L.B. Antonio and M.B. Daniel et al., 2012. Staging of prostate cancer. Histopathol., 60: 87-117. DOI: $10.1111 /$ j.1365-2559.2011.04025.x

Chin, S.P., J.L. Dickinson and A.F. Holloway, 2011. Epigenetic regulation of prostate cancer. Clin. Epigenetics, 2: 151-69. DOI: 10.1007/s13148-011-0041-7

Cho, B., H. Lee, S. Jeong, B. Yung-Jue and J.L. Hyun et al., 2003. Promoter hypomethylation of a novel cancer/testis antigen gene CAGE is correlated with its aberrant expression and is seen in premalignant stage of gastric carcinoma. Biochem. Biophys. Res. Commun., 307: 52-63. DOI:10.1016/S0006-291X(03)01121-5

Cho, N.Y., J.H. Kim, K.C. Moon and G.H. Kang, 2009. Genomic hypomethylation and $\mathrm{CpG}$ island hypermethylation in prostatic intraepithelial neoplasm. Virchows Arch., 454: 17-23. DOI: $10.1007 / \mathrm{s} 00428-008-0706-6$

Costa-Pinheiro, P., H.R. Patel, R. Henrique and C. Jerónimo, 2014. Biomarkers and personalized risk stratification for patients with clinically localized prostate cancer. Expert Rev. Anticancer Ther., 14: 1349-1358. DOI: 10.1586/14737140.2014.952288

Demichelis, F. and J.L. Stanford, 2014. Genetic predisposition to prostate cancer: Update and future perspectives. Urol. Oncol., 33: 75-84. DOI: $10.1016 /$ j.urolonc.2014.04.021

Ehrlich, M., 2009. DNA hypomethylation in cancer cells. Epigenomics, 1: 239-259. DOI:10.2217/epi.09.33

Ellinger, J., K. Haan, L.C. Heukamp, K. Philip and B. Reinhard et al., 2008. CpG island hypermethylation in cell-free serum DNA identifies patients with localized prostate cancer. Prostate, 68: 42-49. DOI: 10.1002/pros.20651

Epstein, J.I., 2010. An update of the Gleason grading system. J. Urol., 183: 433-440. DOI: $10.1016 /$ j.juro.2009.10.046 
Hammoud, S.S., B.R. Cairns and D.A. Jones, 2013. Epigenetic regulation of colon cancer and intestinal stem cells. Curr. Opin. Cell Biol., 25: 177-183. DOI: $10.1016 /$ j.ceb.2013.01.007

Goessl, C., M. Müller, R. Heicappell, K. Hans and M. Kurt et al., 2001. DNA-based detection of prostate cancer in blood, urine and ejaculates. Ann. N.Y. Acad. Sci., 945: 51-58. DOI: 10.1111/j.1749-6632.2001.tb03863.x

Irizarry, R.A., C. Ladd-Acosta, B. Wen, W. Zhijin and M. Carolina et al., 2009. The human colon cancer methylome shows similar hypo- and hypermethylation at conserved tissue-specific $\mathrm{CpG}$ island shores. Nat. Genet., 41: 178-186. DOI: 10.1038/ng.298

Kang, G.H., S. Lee, H.J. Lee and K.S. Hwang, 2004. Aberrant $\mathrm{CpG}$ island hypermethylation of multiple genes in prostate cancer and prostatic intraepithelial neoplasia. J. Pathol., 202: 233-240. DOI: $10.1002 /$ path. 1503

Karam, J.A., Y. Lotan, C.G. Roehrborn, A. Raheela and I.K. Pierre et al., 2007. Caveolin-1 overexpression is associated with aggressive prostate cancer recurrence. Prostate, 67: 614-622. DOI: 10.1002/pros.20557

Karpf, A.R., S. Bai, S.R. James, L.M. James and M.W. Elizabeth et al., 2009. Increased expression of androgen receptor coregulator MAGE-11 in prostate cancer by DNA hypomethylation and cyclic AMP. Mol. Cancer Res., 7: 523-35.

DOI: $10.1158 / 1541-7786 . M C R-08-0400$

Lee, W.H., W.B. Isaacs, G.S. Bova and W.G. Nelson, 1997. CG island methylation changes near the GSTP1 gene in prostatic carcinoma cells detected using the polymerase chain reaction: A new prostate cancer biomarker. Cancer Epidemiol. Biomarkers Prev., 6: 443-450. PMID: 9184779

Li, L.C., P.R. Carroll and R. Dahiya, 2005. Epigenetic changes in prostate cancer: Implication for diagnosis and treatment. J. Natl. Cancer Inst., 97:103-115. DOI: $10.1093 /$ jnci/dji010

Lilja, H., D. Ulmert and A.J. Vickers, 2008. Prostatespecific antigen and prostate cancer: Prediction, detection and monitoring. Nat. Rev. Cancer, 8: 268-728. DOI: $10.1038 / \mathrm{nrc} 2351$

Michaelson, M.D., S.E. Cotter, P.C. Gargollo, L.Z. Anthony and M.D. Douglas et al., 2008. Management of complications of prostate cancer treatment. CA: Cancer J. Clin., 58: 196-213. DOI: $10.3322 /$ CA.2008.0002

Nakayama, T., M. Watanabe, M. Yamanaka, H. Yoshifumi and S. Hiroyoshi et al., 2001. The role of epigenetic modifications in retinoic acid receptor beta2 gene expression in human prostate cancers. Lab. Invest., 81: 1049-1057. PMID: 11454993
Ogishima, T., H. Shiina, J.E. Breault, L. Tabatabai and W.W. Bassett et al., 2005. Increased heparanase expression is caused by promoter hypomethylation and up-regulation of transcriptional factor early growth response-1 in human prostate cancer. Clin. Cancer Res., 11: 1028-1036. PMID: 15709168

Patra, S.K. and S. Bettuzzi, 2007. Epigenetic DNAmethylation regulation of genes coding for lipid raft-associated components: A role for raft proteins in cell transformation and cancer progression (Review). Oncol. Rep., 17: 1279-1290.

DOI: $10.3892 /$ or.17.6.1279

Pulukuri, S.M., S. Patibandla, J. Patel, N. Estes and J.S0 Rao et al., 2007. Epigenetic inactivation of the Tissue Inhibitor of Metalloproteinase-2 (TIMP-2) gene in human prostate tumors. Oncogene, 26: 5229-5237. DOI: $10.1038 /$ sj.onc.1210329

Richiardi, L., V. Fiano, L. Vizzini, D.M. Laura and D. Luisa et al., 2009. Promoter methylation in $A P C$, RUNX3 and GSTP1 and mortality in prostate cancer patients. J. Clin. Oncol., 27: 3161-3168. DOI: $10.1200 /$ JCO.2008.18.2485

Rosenbaum, E., M.O. Hoque, Y. Cohen, Z. Marianna and A.E. Mario et al., 2005. Promoter hypermethylation as an independent prognostic factor for relapse in patients with prostate cancer following radical prostatectomy. Clin. Cancer Res., 11: 8321-8325. DOI: 10.1158/1078-0432.CCR-05-1183

Ross, J.S., P. Kaur, C.E. Sheehan, A.G. F. Hugh and A.K.J. Ronald et al., 2003. Prognostic significance of matrix metalloproteinase 2 and tissue inhibitor of metalloproteinase 2 expression in prostate cancer. Mod. Pathol., 16: 198-205. DOI: 10.1097/01.MP.0000056984.62360.6C

Rouprêt, M., V. Hupertan, J.W. Catto, R.Y. David and R. Ishtiaq et al., 2008. Promoter hypermethylation in circulating blood cells identifies prostate cancer progression. Int. J. Cancer, 122: 952-956. DOI: $10.1002 /$ ijc.23196

Ribarska, T., K-M.M. Bastian, A. Koch and W.A. Schulz., 2012. Specific changes in the expression of imprinted genes in prostate cancer-implications for cancer progression and epigenetic regulation. Asian. J. Androl., 14: 436-450. DOI: 10.1038/aja.2011.160

Tilandyová, P., K. Kajo, J. Kliment, L. Plank and Z. Lasabová et al., 2010. Detection of DNA hypermethylation as a potential biomarker for prostate cancer. Klin. Onkol., 23: 293-299. PMID: 21061679

Woodson, K., K.J. O'Reilly, J.C. Hanson, N. Dayne and L.W. Elyse et al., 2008. The usefulness of the detection of GSTP ${ }_{1}$ methylation in urine as a biomarker in the diagnosis of prostate cancer. J. Urol., 179: 508-512. DOI: 10.1016/j.juro.2007.09.073 
Yang, B., H. Sun, W. Lin, H. Wugang and L. Hang et al., 2013. Evaluation of global DNA hypomethylation in human prostate cancer and prostatic intraepithelial neoplasm tissues by immunohistochemistry. Urol. Oncol., 31: 628-634.

DOI: 10.1016/j.urolonc.2011.05.009

Yegnasubramanian, S., M.C. Haffner, Y. Zhang, G. Bora and C.C. Toby et al., 2008. DNA hypomethylation arises later in prostate cancer progression than $\mathrm{CpG}$ island hypermethylation and contributes to metastatic tumor heterogeneity. Cancer Res., 68: 8954-8967. DOI: 10.1158/0008-5472.CAN-07-6088
Yang, M. and J.Y. Park, 2012. DNA methylation in promoter region as biomarkers in prostate cancer. Methods Mol. Biol., 863: 67-109.

DOI: $10.1007 / 978-1-61779-612-8$ 5

Zon, G., M.A. Barker, P. Kaur, G. Susan and W.J. Lawrence et al., 2009. Formamide as a denaturant for bisulfite conversion of genomic DNA: Bisulfite sequencing of the GSTPi and RARbeta 2 genes of 43 formalin-fixed paraffin-embedded prostate cancer specimens. Anal Biochem 392: 117-125. DOI: 10.1016/j.ab.2009.06.001 